\title{
The Portfolio Decision under the VAR Restriction
}

\author{
Jinwen $\mathrm{Wu}$ \\ School of Economic and Management, South China Normal University, Guangzhou 510006, China \\ E-mail: wujinwen@139.com
}

\begin{abstract}
The VaR, a new appearing financial risk-manage tool, have been applied widely. Many financial setups have accustomed to measure the risk of a portfolio with theVaR. So it is very necessary to discuss the portfolio choice problem under the $V a R$ constraint. In this paper, by setting and solving the portfolio choice model under the VaR constraint, we illustrate that the use of the $V a R$ constraint reduces the array of choice to a more manageable range. The probability of target $V a R$, therefore, can be thought of as a risk tolerance assessment tool (when coupled with another measure of risk).
\end{abstract}

Keywords: The VaR constraint, Portfolio, The optimum weight

\section{Introduction}

In the path-breaking work on Portfolio Selection, Markowitz (1952) developed the concept of an efficient portfolio in terms of the expected return and standard deviation of return (i.e.(E, o) criteria). Modern Portfolio Theory (MPT) has become one of the most important bases in modern capital market, and been applied widely in the practice of investment. In the absence of specific knowledge of investor's preference, however, it cannot be determined which of any two efficient portfolios is better. Baumel (1963) replaced the $(E, \sigma)$ criteria with the $(E, E-k \sigma)$ criteria, where $k$ stands for the investor's attitude toward risk. Baumol demonstrated that his $(E, E-k \sigma)$ criteria yield a smaller efficient set, which is a subset of the Markowitz efficient set, and therefore reduces the range of alternatives from which the investor has to select his portfolio. Recently, Wang Shouyang (1999) studied the portfolio choice model that includes trade cost. This work makes the MPT become more satisfactory to the investor's actual needs. Seeing from mathematical eyes, the classical Markowitz model and its plasmodium are some quadratic programmed models with regard to defined matrix.

On the other hand, Value at Risk ( $V a R)$ is an important measure of exposure of a given portfolio of securities to different kinds of risk inherent in financial environment. By now, it became a tool for risk management in financial industry and part of industrial regulatory mechanisms. Meanwhile Basel Committee requested financial setups to measure the market risk with VaR. Considerable amount of research was dedicated recently to development of methods of risk manage-ment based on Value at Risk. This literature is dedicated mainly to efficient techniques for computing VaR of a given portfolio. To construct the portfolio under the thought of $V a R$, therefore, is one of the most important problems that the international investment market is being faced.

In this paper we combine the notion of VaR with portfolio optimality. More precisely, we seek to define portfolio that produces maximal yield (or minimum risk) and at the same time satisfies constraints on Value at Risk. Our aim is to develop a theory that is the Markovitz theory for optimal mean-variance portfolios in VaR constraint and provide algorithmic tools for computing such portfolios. Our emphasis here is on algorithms because, unlike classical optimal mean-variance portfolios, optimal mean-variance portfolios in VaR constraint generally defy analysis with simple analytical tools.

\section{Building and explaining of the model}

As well known, the classical Markowitz model is:

$$
\left\{\begin{array}{l}
\min \sigma_{p}^{2}=X^{T} \Sigma X \\
\max E\left(r_{p}\right)=X^{T} R \\
\text { s.t. } \sum_{i=1}^{n} x_{i}=1
\end{array}\right.
$$

here, $R=\left[R_{1}, R_{2}, \Lambda R_{n}\right]^{T}, R_{i}=E\left(r_{i}\right)$ is the expected return rate of the $i$ th asset. $X=\left[x_{1}, x_{2}, \Lambda, x_{n}\right]^{T}$ is the weights vector of portfolio. $\Sigma=\left[\sigma_{i j}\right]_{n \times n}$ is the covariance matrix of $n$ assets. $R_{p}=E\left(r_{p}\right)$ and $\sigma_{p}^{2}$ are the expected return 
rate and its variance of portfolio. The $\sigma_{p}$, which is used to measure the risk of portfolio by Markowitz and called the standard deviation of the return rate, express the scope between the return rate of portfolio $r_{p}$ and $E\left(r_{p}\right)$.

It is common knowledge that the solution of the model $(I)$ is the curve $\overrightarrow{A B}$ in $\sigma_{p}-R_{p}$ space (figure 1) and is called the efficient frontier of portfolio.

Assessing risk of portfolio as the probability of failing to earn a minimum return, we call the way as the VaR risk-measure way. The minimum return is called $V a R$ of the portfolio. This can be denoted:

$\operatorname{Pr}\left(r_{p}<-V a R\right) \leq \alpha$

here $\alpha$ is a given probability.

Supposing the distribution of portfolio is statistics normal distribution, we incorporate (2.1) into the model $(I)$. Then we get the portfolio model in VaR constraint:

$$
\begin{array}{ll}
\min & \sigma_{p}^{2}=X^{T} \Sigma X \\
\max & E\left(r_{p}\right)=X^{T} R \\
& \operatorname{Pr}\left(r_{p}<-V a R\right) \leq \alpha \\
\text { s.t. } & \sum_{i=1}^{n} x_{i}=1
\end{array}
$$

Because (2.1) can be taken the place of

$$
\operatorname{VaR}=-\left[E\left(r_{p}\right)-\Phi^{-1}(\alpha) \sigma_{p}\right]
$$

here $\Phi(\bullet)$ is the standard normal distribution function. The solution of model (II) is the pitch arc $\overline{A B}$ in $\sigma_{p}-R_{p}$ space (Figure 2). The pitch arc is called the efficient frontier of portfolio in VaR constraint.

Figure 2 is a risk-return diagram illustrating that the $\operatorname{VaR}$ constraint plots as a straight line with slope $\Phi^{-1}(\alpha)$ and Y-axis intercept of $-\mathrm{VaR}$. All portfolios lying on or above this line have a $1-\alpha$ probability of exceeding the $-\mathrm{VaR}$ return minimum, while portfolios that lie below this line will not exceed a return of - VaR with $1-\alpha$ confidence. As one would expect, the minimum return for a given confidence level will increase as the expected return increases (holding the standard deviation constant) and will decreases as the standard deviation increases (holding the expected return constant).

Assessing risk as the probability of failing to earn a minimum return can further be helpful in selecting the optimal portfolio from an array of portfolios representing the efficient frontier. This is because the VaR restriction essentially provides information regarding the risk tolerance of the investor. By formally introducing the $\operatorname{VaR}$ as a constraint into the portfolio optimization problem, the range of choice across the full array of efficient portfolios can be narrowed to those that are most relevant to the investor. That is, the constraint allows the investor to focus on that segment of the frontier that best fits his or her degree of risk aversion. The probability of target VaR, therefore, can be thought of as a risk tolerance assessment tool (when coupled with another measure of risk).

Figure 2 illustrates this process of selecting an optimal portfolio in conjunction with a VaR constraint. It shows an efficient frontier of portfolios along with the $V a R$ constraint of a $1-\alpha$ probability of exceeding a $-V a R$ return. Note again that the VaR constraint segments the combination of expected returns and risk. Portfolios to the right of the line violate the constraint; their realized returns do not exceed $-V a R$ with $1-\alpha$ confidence. Portfolios to the right of the line actually satisfy a higher $1-\alpha$ confidence limit than-VaR .

At the same time, only portfolio risk-return trade-offs on or below the efficient frontier are feasible. As Figure 2 shows, the set of feasible portfolios satisfying a VaR constraint is limited. The set of portfolios to be considered for selection in the optimization decision is limited to the shaded area of portfolios that both are feasible and also satisfy the specified $V a R$ constraint. In this case, the investor would select a portfolio from the segment ranging from point A to B, as these represent the optimal portfolios available. In this illustration, and in other cases, the use of the VaR constraint reduces the array of choice to a more manageable range. We should note, however, that the constraint may be either too severe, so as to preclude the possibility of any choice, or too loose, so as to maintain much, if not all, of the efficient frontier as a range of choice.

\section{Solving of the model}

Knowing from figure 2, we only need to find the weights of point A and B. To solve the weights of the two points, however, won't work with the simple Laganarge algorithms. So we will solve the problem with a geometric algorithm that was produced by us. 


\subsection{Introducing of the geometric algorithm}

We consider a finite set of assets $i=1,2, \Lambda, n$ which can be any kind of financial assets, stocks, bonds and options being the most common examples. Weight vector of Portfolio $X$ is characterized by positions in these assets:

$$
X=\left(x_{1}, x_{2}, \Lambda, x_{n}\right)
$$

Because $x_{1}+x_{2}+\Lambda+x_{n}=1, \quad x_{n}=1-x_{1}-x_{2}-\Lambda-x_{n-1}$. The expected return $R_{p}=E\left(r_{p}\right)$ and its variance risk $\sigma_{p}^{2}$ of portfolio can be repressed:

$$
\begin{aligned}
R_{p}= & x_{1} R_{1}+x_{2} R_{2}+\Lambda+x_{n-1} R_{n-1}+\left(1-x_{1}-\Lambda-x_{n-1}\right) R_{n} \\
\sigma_{p}^{2}= & x_{1}^{2} \sigma_{11}+x_{2}^{2} \sigma_{22}+\Lambda+x_{n-1}^{2} \sigma_{n-1 n-1}+\left(1-x_{1}-\Lambda-x_{n-1}\right)^{2} \sigma_{n n} \\
& +2 x_{1} x_{2} \sigma_{12}+\Lambda 2 x_{1} x_{n-1} \sigma_{1 n-1}+2 x_{1}\left(1-x_{1}-\Lambda-x_{n-1}\right) \sigma_{1 n} \\
& +\Lambda+2 x_{n-1}\left(1-x_{1}-\Lambda-x_{n-1}\right) \sigma_{n-1 n}
\end{aligned}
$$

The covariance matrix $\Sigma$ is a definite matrix. So the formula (3.2) stands for a equal-variance ellipsoid in the weights-space $\left(x_{1}, x_{2}, \Lambda x_{n-1}\right)$. With regard to different $\sigma_{p}^{2}$, we can get a family of equal-variance ellipsoids that have a concentric MVP. The center MVP expresses the portfolio that has the least risk in the set of feasible portfolios. In the weights-space $\left(x_{1}, x_{2}, \Lambda x_{n-1}\right)$, the formula (3.1) stands for a equal-expected-return super-plane. With regard to different $R_{p}$, we can obtain a family of parallel super-plane. So the optimal weights of the portfolio that includes $n$ assets should be the tangential point of the equal-expected-return super-plane (3.1) and the equal-variance ellipsoid (3.2). Joining these tangential points, we can obtain a straight line that is called the critical-line of the portfolio of $n$ assets. In fact, the critical-line is the manifestation of the efficient frontier in weights-space.

Knowing from differential geometry, the normal vector of the formula (3.1) in point $\left(x_{1}, x_{2}, \Lambda x_{n-1}\right)$ is:

$$
\left(R_{1}-R_{n}, R_{2}-R_{n}, \Lambda, R_{n-1}-R_{n}\right)
$$

The normal vector of the formula (3.2) in point $\left(x_{1}, x_{2}, \Lambda x_{n-1}\right)$ is

$$
\left(\left(\sigma_{11}+\sigma_{n n}-2 \sigma_{1 n}\right) x_{1}+\Lambda+\left(\sigma_{1 k}+\sigma_{n n}-\sigma_{1 n}-\sigma_{k n}\right) x_{k}+\Lambda+\left(\sigma_{1 n-1}+\sigma_{n n}-\sigma_{1 n}-\sigma_{n-1, n}\right) x_{n-1}+\sigma_{1 n}-\sigma_{n n},\right.
$$

$\Lambda \Lambda \Lambda \Lambda \Lambda \Lambda$,

$$
\left(\sigma_{1 k}+\sigma_{n n}-\sigma_{1 n}-\sigma_{k n}\right) x_{11}+\Lambda+\left(\sigma_{k k}+\sigma_{n n}-2 \sigma_{k n}\right) x_{k}+\Lambda+\left(\sigma_{k, n-1}+\sigma_{n n}-\sigma_{k n}-\sigma_{n-1, n}\right) x_{n-1}+\sigma_{k n}-\sigma_{n n}
$$

$\Lambda \Lambda \Lambda \Lambda \Lambda \Lambda$,

$$
\left.\left(\sigma_{1, n-1}+\sigma_{n n}-\sigma_{1 n}-\sigma_{n-1, n}\right) x_{1}+\Lambda+\left(\sigma_{k, n-1}+\sigma_{n n}-\sigma_{k n}-\sigma_{n-1, n}\right) x_{k}+\Lambda+\left(\sigma_{n-1, n-1}+\sigma_{n n}-2 \sigma_{n-1, n}\right) x_{n-1}+\sigma_{n-1, n}-\sigma_{n n}\right)
$$

Let

$$
\begin{aligned}
& P_{1}=[1,0,0, \Lambda, 0,0,-1] \\
& P_{2}=[0,1,0, \Lambda, 0,0,-1] \\
& \Lambda \Lambda \Lambda \Lambda \Lambda \Lambda \\
& P_{n-1}=[0,0,0, \Lambda, 0,1,-1]
\end{aligned}
$$

$$
Q=\left[\begin{array}{lllll}
1 & 0 & \Lambda & 0 & 0 \\
0 & 1 & \Lambda & 0 & 0 \\
M & M & \mathrm{O} & \mathrm{M} & \mathrm{M} \\
0 & 0 & \Lambda & 1 & 0 \\
-1 & -1 & \Lambda & -1 & 1
\end{array}\right]
$$$$
W=\left[\begin{array}{l}
x_{1} \\
x_{2} \\
\mathrm{M} \\
x_{n-1} \\
1
\end{array}\right]
$$

Then the normal vector of the formula (3.1) in point $\left(x_{1}, x_{2}, \Lambda x_{n-1}\right)$ can be simplified to

$$
\left(P_{1} \Sigma Q W, P_{2} \Sigma Q W, \Lambda, P_{k} \Sigma Q W, \Lambda, P_{n-1} \Sigma Q W\right)
$$

According to the definition of the critical-line, we can obtain the equation of the critical-line that is

$$
\frac{P_{1} \Sigma Q W}{R_{1}-R_{n}}=\frac{P_{2} \Sigma Q W}{R_{2}-R_{n}}=\Lambda=\frac{P_{k} \Sigma Q W}{R_{k}-R_{n}}=\Lambda=\frac{P_{n-1} \Sigma Q W}{R_{n-1}-R_{n}}
$$

By the formula (2.3), we can obtain a linear equations group that is composed of $n-2$ linear equations: 


$$
\left\{\begin{array}{l}
a_{11} x_{1}+a_{12} x_{2}+\Lambda+a_{1, n-1} x_{n-1}=b_{1} \\
a_{21} x_{1}+a_{22} x_{2}+\Lambda+a_{2, n-1} x_{n-1}=b_{2} \\
\Lambda \Lambda \Lambda \Lambda \Lambda \Lambda \\
a_{n-2,1} x_{1}+a_{n-2,2} x_{2}+\Lambda+a_{n-2, n-1} x_{n-1}=b_{n-2}
\end{array}\right.
$$

Here

$$
\begin{aligned}
& a_{i j}=\frac{\sigma_{i j}+\sigma_{n n}-\sigma_{i n}-\sigma_{j n}}{R_{i}-R_{n}}-\frac{\sigma_{j, n-1}+\sigma_{n n}-\sigma_{j n}-\sigma_{n-1, n}}{R_{n-1}-R_{n}} \\
& b_{i}=-\frac{\sigma_{i n}-\sigma_{n n}}{R_{i}-R_{n}}+\frac{\sigma_{n-1, n}-\sigma_{n n}}{R_{n-1}-R_{n}} \\
& (i=1,2, \Lambda, n-2, j=1,2, \Lambda, n-1)
\end{aligned}
$$

\subsection{The solution of the portfolio based on the geometric way}

The formula (2.6) can be turn into the following form:

$$
\sigma_{p}^{2}=\frac{1}{\left(\Phi^{-1}(\alpha)\right)^{2}}\left(R_{p}^{2}+2 \operatorname{VaRR}_{p}+\operatorname{VaR}^{2}\right)
$$

Taking the formula (2.2) and (2.3) into (3.5), we can get:

$$
X^{T} \Sigma X=\frac{1}{\left(\Phi^{-1}(\alpha)\right)^{2}}\left[\operatorname{VaR}^{2}+2 \operatorname{VaR} X^{T} R+\left(X^{T} R\right)^{2}\right]
$$

The rank of the linear equations group is $n-2$, so the number of its based solutions system is 1 . That is saying that $x_{2}, x_{3}, \Lambda x_{n-1}$ can all be expressed by $x_{1}$. Knowing from the formula (2.5), therefore, $x_{n}$ can be expressed by $x_{1}$ too. Taking $x_{1}, x_{2}, \Lambda x_{n}$ into the formula (3.6), we can get a quadratic equation with one unknown concerning $x_{1}$. According to the extract roots formula, we can find the two roots of $x_{1}$. Then we can get naturally the value of $x_{2}, x_{3}, \Lambda x_{n}$. Noting that $x_{1}$ has two roots, so there are two groups of solutions that are the weights of point A and $\mathrm{B}$ respectively. According to the following formulas:

$$
\begin{array}{r}
R_{p}=X^{T} R \\
\sigma_{p}^{2}=X^{T} \Sigma X
\end{array}
$$

We can obtain the expected returns $R_{A}$ and $R_{B}$ of the portfolios, and their variances $\sigma_{A}^{2}$ and $\sigma_{B}^{2}$ at points A and B respectively.

Thus we can obtain the range of portfolio choice in $V a R$ constraint:

$$
R_{B} \leq R_{P} \leq R_{A}, \quad \sigma_{B}^{2} \leq \sigma_{p}^{2} \leq \sigma_{A}^{2}
$$

Given an expected return $R_{p}$ of portfolio within the range, we can find the optimal portfolio weights in the critical-line by connecting (3.1) and (3.4). The variance of portfolio will reduce to minimum under the optimal portfolio weights. This process can be finished through decreasing unknowns of linear equations group. The minimum variance of portfolio can be obtained by the formula (3.2). Given a variance $\sigma_{p}^{2}$ of portfolio within the range, at the same time, we can find the optimal portfolio weights in the critical-line by connecting (3.2) and (3.4). The expected return of portfolio will increase to maximum under the optimal portfolio weights. The maximum expected return of portfolio could be obtained by the formula (3.2).

We should note, however, that the $\operatorname{VaR}$ constraint may be either too severe, so as to find non-solution of the quadratic equation with one unknown concerning $x_{1}$, and preclude the possibility of any choice. The VaR constraint, of course, may be lead to find the sole solution of the quadratic equation with one unknown concerning $X_{1}$, and lead to take the choice of portfolio is sole too.

\section{Example}

To demonstrate how the portfolio model in VaR constraint is applied in asset allocation, we will consider three major asset classes: common stocks, long-term bonds, and money market instruments. These are the securities commonly used 
by portfolio managers and major investors, either as a complete list of classes for consideration or as essential classes within more extended groupings of asset classes. These can thus be representative of the sort of actual results produced from an asset allocation while at the same time being clear enough in application to illustrate the process.

Table 1 shows the realized return and standard deviation of return for each of the three asset classes, as well as the correlation across those asset classes shown over the 129-1993 period.

Knowing from Table $1, R=\left[\begin{array}{lll}12.3 & 5.4 & 3.7\end{array}\right]^{T}$, and

$$
\begin{aligned}
& \sigma_{11}=\sigma_{1}^{2}=20.5^{2}=420.25 \quad \sigma_{22}=\sigma_{2}^{2}=8.7^{2}=75.69 \quad \sigma_{33}=\sigma_{3}^{2}=3.3^{2}=10.89 \\
& \sigma_{12}=\sigma_{21}=\sigma_{1} \sigma_{2} \rho_{12}=20.3319 \sigma_{13}=\sigma_{31}=\sigma_{1} \sigma_{3} \rho_{13}=-33.825 \quad \sigma_{23}=\sigma_{32}=\sigma_{2} \sigma_{3} \rho_{23}=6.8904 \\
& \Sigma=\left[\begin{array}{lll}
420.250 & 20.3319 & -33.825 \\
20.3319 & 75.6900 & 6.8904 \\
-33.825 & 6.89040 & 10.89
\end{array}\right]
\end{aligned}
$$

i.e.

So the linear equations group is turned into:

$$
22.4738 x_{1}-36.0607 x_{2}=2.8467
$$

Therefore

$$
\begin{aligned}
& x_{2}=-0.07894+0.6232 x_{1} \\
& x_{3}=1-x_{1}-x_{2}=1.07894-1.6232 x_{1}
\end{aligned}
$$

Supposing $\operatorname{VaR}=5.0 \%, \alpha=5 \%$, then $\Phi^{-1}(\alpha)=1.65$. So the formula (3.3) is turned into:

$$
X^{T} \Sigma X=\frac{1}{\left(\Phi^{-1}(\alpha)\right)^{2}}\left[\operatorname{VaR}^{2}+2 \operatorname{VaR} X^{T} R+\left(X^{T} R\right)^{2}\right]=\frac{1}{2.7225}\left[25+10 X^{T} R+\left(X^{T} R\right)^{2}\right]
$$

Taking $R, \Sigma$ and

$$
X=\left[x_{1}, x_{2}, x_{3}\right]^{T}=\left[x_{1}, \quad-0.1267+0.6223 x_{1}, \quad 1.1267-1.6223 x_{1}\right]^{T}
$$

into (4.2), we can get the quadratic equation with one unknown concerning $x_{1}$ :

$$
565.1028 x_{1}^{2}-171.5078 x_{1}-14.9769=0
$$

According to the extract roots formula, we can find the two roots of $x_{1}$ :

$$
x_{1}=0.3743 \text { or } x_{1}=-0.0708
$$

Taking the value of $x_{1}$ into the expressed formulas $x_{2}$ and $x_{3}$, we can obtain the portfolio weights at points A and B:

$$
\begin{aligned}
& x_{1}^{A}=0.3743 \quad x_{2}^{A}=0.1543 \quad x_{3}^{A}=0.4716 \\
& x_{1}^{B}=-0.0708 \quad x_{1}^{B}=-0.123 \quad x_{1}^{B}=1.1938
\end{aligned}
$$

According to the formulas (3.4) and (3.5), the expected returns and their variances at points A and B are respectively:

$$
\begin{array}{ll}
R_{A}=7.182 \% & R_{B}=2.882 \% \\
\sigma_{A}^{2}=54.511 & \sigma_{B}^{2}=20.8834
\end{array}
$$

So the standard deviations of portfolio at points $\mathrm{A}$ and $\mathrm{B}$ are respectively:

$$
\sigma_{A}=7.383 \% \quad \sigma_{B}=4.57 \%
$$

Thus we can obtain the range of portfolio choice in our $V a R$ constraint:

$2.882 \% \leq R_{P} \leq 7.182 \%, \quad 4.57 \% \leq \sigma_{P} \leq 7.383 \%$

All portfolios lying in the range have a 95 percent probability of returns exceeding

$$
E\left(r_{p}\right)-1.65 \sigma_{P}
$$

That is saying that there is only a $5 \%$ probability of a risk-loss exceeding $V a R=5.0 \%$.

Given an expected return $R_{p}$ of portfolio within the range, we can find the optimal portfolio weights directly by connecting (3.1) and (4.1). Given a variance $\sigma_{p}^{2}$ of portfolio within the range, on the other hand, we can find the optimal portfolio weights directly by connecting (3.2) and (4.1). 
Given $R_{P}=7 \%$ for example, the formula (3.1) is changed into

$$
12.3 x_{1}+5.4 x_{2}+3.7\left(1-x_{1}-x_{2}\right)=7
$$

i.e.

$$
8.6 x_{1}+1.7 x_{2}=3.3
$$

Then the formula (4.1) is turned into:

$$
22.4738 x_{1}-36.0607 x_{2}=2.8467
$$

To solve the equations group that is formed of the two equations (4.4) and (4.5), we can get:

$$
x_{1}=0.3555 \quad x_{2}=0.1428
$$

So

$$
x_{3}=1-x_{1}-x_{2}=0.5017
$$

According to the formula (3.8), the minimum variance of portfolio is:

$$
\sigma_{p}^{2}=X^{T} \Sigma X=\left[\begin{array}{lll}
0.3555 & 0.1428 & 0.5017
\end{array}\right]\left[\begin{array}{lll}
420.250 & 20.3319 & -33.825 \\
20.3319 & 75.6900 & 6.8904 \\
-33.825 & 6.89040 & 10.89
\end{array}\right]\left[\begin{array}{l}
0.3555 \\
0.1428 \\
0.5017
\end{array}\right]=48.3818
$$

Therefore, the minimum standard deviation of portfolio is

$$
\sigma_{p}=6.9557 \%
$$

Given a standard deviation of portfolio $\sigma_{p}$, whereas, we can find the optimal portfolio weights by imitating the solving process of the equation (4.2) (substituting $\sigma_{p}^{2}$ for $\frac{1}{\left(\Phi^{-1}(\alpha)\right)^{2}}\left[\operatorname{VaR}^{2}+2 \operatorname{VaRX}{ }^{T} R+\left(X^{T} R\right)^{2}\right]$ in the right hand of the formula (4.2)). Noting there are two groups of the optimal portfolio weights. Next computing their $R_{p} \mathrm{~s}$ with the formula (3.7), we can obtain the real optimal portfolio weights by comparing. We don't explain the process in detail.

\section{References}

A.A. Gaivoronski, E. Messina, and A. Sciomachen. (1994). A statistical generalized programming algorithm for stochastic optimization problems. Annals of Operations Research.

Best.M. and R.Grauer. (1990). The Sensitivity of Mean-Variance Efficient Portfolios to Changes in Asset Means: Some Analytical and Comutational Results, Review of Financial Studies.

Chopra.V., and W.Ziemba. (1993). The Effect of Eorror in Means, Variances, and Covariance on Optimal Portfolio Choice. Journal of Portfolio Management. 19, 6-11.

Chow, G. (1995). Portfolio selection based on return, risk and relative performance. Financial Analysis Journal. 2, $54 \sim 60$.

C.O Alexander and C.T. Leigh. (1997). On the covariance matrices used in value at risk models. The Journal of Derivatives. 11, 50-62.

Duffie, D., Pan, J. (1997). An Overview of Value at Risk. Journal of Derivatives. 3, 7 49.

Fong, M.G., Lin, K. (1999). A New Analytical Approach to VaR. Journal of Portfolio Management, 25 Anniversary issue , May, 88 97.

Gibbons. M., S. Ross, and J. Shanken. (1989). Test of the Efficiency of a Given Portfolio. Econometric. 57, 11121-1152. Glyn Holton. (1998). Simulating value-at-risk. Risk, May, 60-63.

Green. R., and B. Hollified. (1992). When Will Mean-Variance Efficient Portfolios Be Well Diversified? Journal of Finance. 47, 1785-1809.

Harry. (1952). Markowitz, Portfolio Selection. Journal of Finance. March, 77-91.

Ibbotson, Roger. G., and Rex A. (1977). Sinquefeld, Stocks, Bonds, Bills, and Inflation: The Past (1926-1976) and The Future (1977-2000), Financial Analysts Research Foundation, Charlottesville, VA.

J. L. Higle and S. Sen. (1991). Stochastic decomposition: An algorithm for stage linear programs with recourse. Mathematics of Operations Research. 16, 650-669.

Lewis, Alan. (1988). A Simple Algorithm for the Portfolio Selection Problem. Journal of Finance.March, 71-82.

Liebowitz, Martin, and Roy Henriksson. (1989). Portfolio Optimization with Shortfall Constraints: A Confidence-Limit 
Approach to Managing Downside Risk. Financial Analysts Journal. March-April. 34-41.

Linsmeier. T.J., Pearson. N.D. (2000). Value at Risk, Financial Analysts Journal. March-April. 47 67.

Manoj K. Singh. (Fall 1997). Value at risk using principal components analysis. The Journal of Portfolio Management. 101-112.

Merton. R.C. (1972). An Analytic Derivation of the Efficient Portfolio Frontier. Journal of Financial and Quantitative Analysis 7.1851-1872.

Michaud. R. (1989). The Markowitz Optimization Enigma: Is Optimized Optimal? Financial Analysts Journal. $31-42$.

Philippe Jorion. (1996). Risk: Measuring the risk in value at risk. Financial Analysts Journal, November-December. 101-112.

Thomas S.Y. Ho, Michael Z.H. Chen, and Fred H.T. Eng. (Fall 1996). VAR analytics: Portfolio struc-ture, key rate convexities, and VAR betas. The Journal of Portfolio Management. 89-98.

Yu. Ermoliev and A. A. Gaivoronski. (1992). Stochastic Quasi-gradient Methods for Optimization of Discrete Event Systems. Annals of Operations Research. 39:1-39.

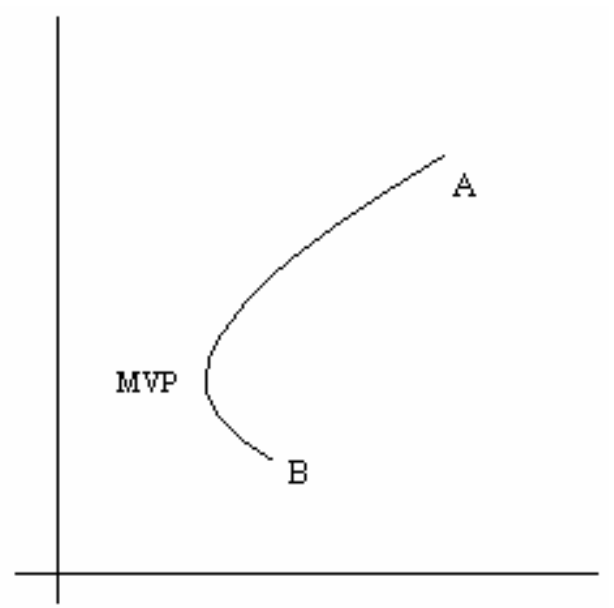

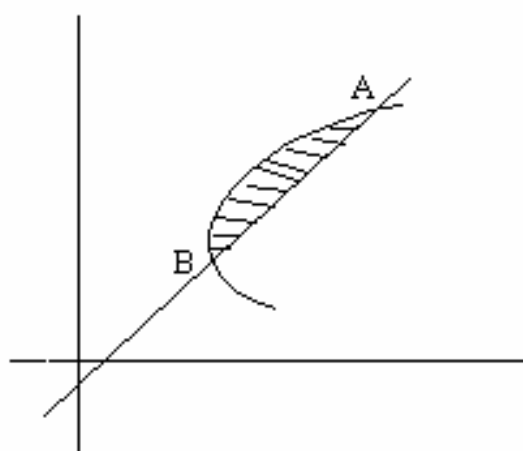

Figure 2.

Figure 1.

Table 1.

Risk-return date (1926-1993)

\begin{tabular}{|c|c|c|c|c|c|}
\hline Asset classes & Mean returns (\%) & Standard deviation (\%) & \multicolumn{3}{|c|}{ Correlation } \\
\hline Common stock & 12.3 & 20.5 & 1.0 & 0.114 & -0.5 \\
\hline Long-term bonds & 5.4 & 8.7 & 0.114 & 1.0 & 0.24 \\
\hline Treasury bills & 3.7 & 3.3 & -0.5 & 0.24 & 1.0 \\
\hline
\end{tabular}

